Radial and Nonradial Pulsations as Probes of Stellar Physics

ASP Conference Series, Vol. 259, 2002

C. Aerts, T.R. Bedding, \& J. Christensen-Dalsgaard, eds.

\title{
Using SX Phœnicis Variables in M55 and Theoretical Period-Luminosity(-Colour-Metallicity) Relations for Distance Determination
}

\author{
J.O. Petersen \\ Astronomical Observatory, NBIfAPG, Copenhagen, Denmark \\ L.M. Freyhammer \\ Royal Observatory of Belgium and University of Brussels (VUB), \\ Brussels, Belgium
}

\begin{abstract}
Theoretical period-luminosity and period-luminosity-colourmetallicity relations are used to give distance estimates for the globular cluster M55 from detailed data for 24 SX Phœnicis variables published recently by Pych et al. (2001). With accurate determinations of effective temperatures for the SX Phe stars application of period-luminositycolour-metallicity relations will lead to improved distances and more secure mode identifications.
\end{abstract}

Very recently Pych et al. (2001, in the following P01) presented detailed CCD photometry of 24 SX Phœnicis variables in the globular cluster M55 and estimated the apparent distance modulus of M55 to be $(m-M)_{V}=13.86 \pm$ 0.25 mag. Petersen \& Christensen-Dalsgaard (1999, PCD99) used theoretical stellar evolution sequences with simultaneous pulsation calculations to derive period-luminosity relations (PLR in the following) and period-luminosity-colourmetallicity relations (PLCZR) for Population I chemical compositions. Here we use similar PLR and PLCZR derived for Population II compositions, in combination with the P01 data for M55, to determine new distance estimates.

To represent the Pop. II SX Phe variables, we select 309 individual models located within the instability strip from 21 theoretical stellar evolution sequences with metal contents in the range $Z=0.0001$ to 0.005 , kindly supplied by J. Christensen-Dalsgaard. Following PCD99, we derive PL relations by leastsquares fits to the model data. Using the period variable $p=\log \Pi_{0}+1.10$, we obtain for the simple PLR fit:

$$
M_{b o l}=-2.922 p+2.099,
$$

with intrinsic scatter $\sigma= \pm 0.174$ mag. Using also $t=\log T_{\text {eff }}-3.875$ and $f=[\mathrm{Fe} / \mathrm{H}]+1.40$, the full PLCZR becomes:

$$
M_{\text {bol }}=-3.765 p+2.138-12.224 t-0.114 f
$$

with intrinsic scatter $\sigma= \pm 0.021$ mag. Thus the introduction of colour and metallicity terms improves the intrinsic scatter in the fitting formula by a factor of more than eight. The intrinsic scatter gives an estimate of the accuracy 
of a derived $M_{b o l}$ which will be obtained from one star with very accurate observational data. Therefore application of PLCZR rather than PLR gives the possibility of very accurate determinations of $M_{b o l}$. The price to pay is that we now need to know the effective temperature to a high accuracy and also a good estimate of the metallicity.

For distance determinations the mode identification is important. P01 select five stars as first overtone variables (1OT in the following) and consider the other stars as fundamental mode (F) oscillators. We select ten $1 \mathrm{OT}$ variables, in addition to those given by $\mathrm{P} 01$ also \# 22,26, 32, 33 and 42. This choice is justified by means of the PLCZR (see below).

Using standard methods to compare the observed periods and $V$-data with the simple PLR, our estimate for the apparent distance modulus becomes $(m-$ $M)_{V}=14.03 \pm 0.21$, where by far the largest contribution to the standard error arises from the intrinsic scatter in the theoretical PLR $( \pm 0.17)$. P01 based their distance estimate on the Hipparcos data for the proto-type variable SX Phe itself, and obtained $(m-M)_{V}=13.86 \pm 0.25 \mathrm{mag}$. The difference of $0.17 \mathrm{mag}$ between these two estimates based on precisely the same observations, but using different methodologies, is less than the $1 \sigma$ errors.

With $[\mathrm{Fe} / \mathrm{H}]=-1.81$ for M55 our PLCZR Eq. (2) can be written

$$
M_{b o l}-c t=-3.765 \log \Pi_{0}-1.957
$$

with $c t=-12.24\left(\log T_{\text {eff }}-3.875\right)$. The critical quantity here is $\log T_{\text {eff }}$, which we determine for each observed SX Phe star from $(B-V)$. Then we can calculate $M_{b o l}-c t$ and compare the sample of SX Phe stars in M55 with Eq. (3). We obtain the best solution by selection of ten of the stars as first overtone variables (while P01 selected five), and determine from the PLCZR: $(m-M)_{V}=13.55 \pm$ 0.13 mag. Here the uncertainty arises from the uncertain observational data, while the contribution from the intrinsic scatter of the PLCZR is negligible ( $\pm 0.02 \mathrm{mag})$.

The difference between this estimate and the value 13.86 mag of P01 is just within the $1 \sigma$ errors. But the difference of $0.48 \mathrm{mag}$ between this value and the result $14.03 \mathrm{mag}$ from the simple PLR is larger than expected, because both estimates are based on the same observational data and (in the end) also on the same theoretical models. A probable explanation is a small systematic error in our derived values of $\log T_{\text {eff }}$.

Ideally, the stars should be located on two parallel lines in the $\left(\log \Pi_{0}, M_{b o l}\right.$ $c t$ ) diagram corresponding to $\mathrm{F}$ and $1 \mathrm{OT}$ oscillators. With sufficiently accurate observations we should find a scatter of only $\pm 0.02 \mathrm{mag}$ around the theoretical lines. Clearly this method with accurate data could give a much safer mode identification from such PLCZ diagrams than earlier applications of PL diagrams have provided.

\section{References}

Petersen, J.O. \& Christensen-Dalsgaard, J. 1999, A\&A, 352, 547

Pych, W., Kaluzny, J., Krzeminski, W., Schwarzenberg-Czerny, A., \& Thompson, I.B. 2001, A\&A, 367, 148 


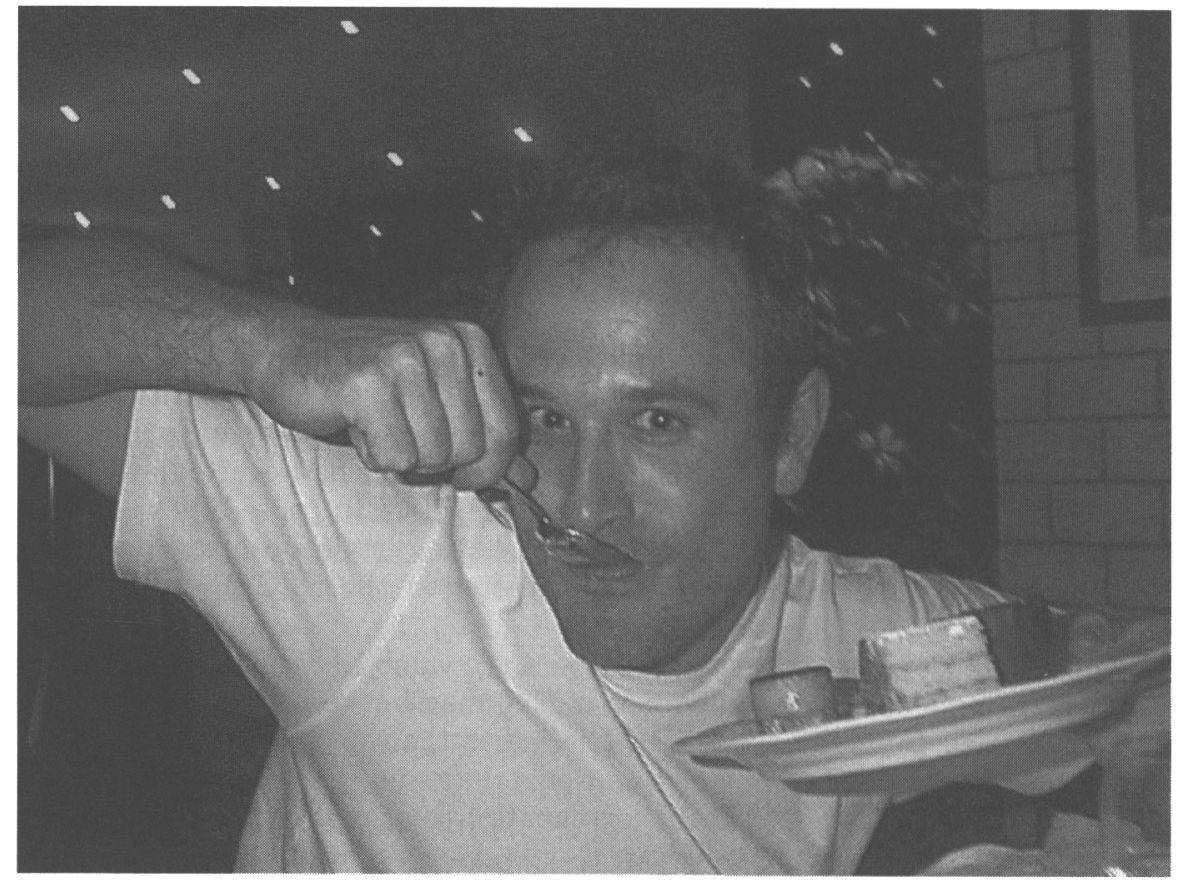

It is Richard Townsend's first participation to a pulsation conference. Evidently, it made him very very hungry. 